\title{
Rapid $\mathrm{Ca}^{2+}$-dependent decrease of protein ubiquitination at synapses
}

\author{
Hong Chen ${ }^{\dagger}$, Simona Polo ${ }^{\ddagger}$, Pier Paolo Di Fiore ${ }^{\ddagger}$, and Pietro V. De Camilli ${ }^{\dagger}$
}

${ }^{\dagger}$ Howard Hughes Medical Institute and Department of Cell Biology, Yale University School of Medicine, New Haven, CT 06510; ${ }^{\ddagger}$ European Institute of

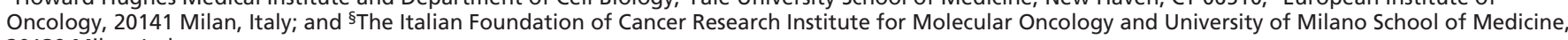
20139 Milan, Italy

Contributed by Pietro V. De Camilli, October 13, 2003

Protein ubiquitination has been implicated in the regulation of axonal growth and synaptic plasticity as well as in the pathogenesis of neurodegenerative diseases. Here we show that depolarization-dependent $\mathrm{Ca}^{2+}$ influx into synaptosomes produces a global, rapid (range of seconds), and reversible decrease of the ubiquitinated state of proteins, which correlates with the $\mathrm{Ca}^{2+}$ dependent dephosphorylation of several synaptic proteins. A similar general decrease in protein ubiquitination was observed in nonneuronal cells on $\mathrm{Ca}^{2+}$ entry induced by ionomycin. Both in synaptosomes and in nonneuronal cells, this decrease was blocked by FK506 (a calcineurin antagonist). Proteins whose ubiquitinated state was decreased include epsin 1, a substrate for the deubiquitinating enzyme fat facets/FAM, which we show here to be concentrated at synapses. These results reveal a fast regulated turnover of protein ubiquitination. In nerve terminals, protein ubiquitination may play a role both in the regulation of synaptic function, including vesicle traffic, and in the coordination of protein turnover with synaptic use.

Covalent conjugation of ubiquitin to proteins represents an important mechanism to regulate their turnover, subcellular distribution, or function. A well established role of protein polyubiquitination is to target cytosolic proteins for degradation in proteasomes (1-3). Conversely, mono- or oligoubiquitination of membrane proteins targets them to multivesicular bodies and eventually to degradation in lyososomes (4). In addition, reversible mono- and oligoubiquitination of proteins are important regulatory mechanisms in a variety of cellular processes $(5,6)$.

Several synaptic proteins undergo ubiquitination (7-11), and this modification has been implicated in the regulation of both pre- and postsynaptic plasticity (11-14). Furthermore, axon guidance defects or aberrant synaptic morphology and function have been associated with abnormal protein ubiquitination or proteasomal degradation (15-19). Malfunction of protein ubiquitination has been implicated in Parkinson's disease (20), and neuronal cytoplasmic inclusions characteristic of neurodegenerative diseases contain ubiquitinated proteins $(9,21,22)$, underscoring the importance of normal ubiquitination metabolism in the nervous system.

Despite evidence for the occurrence of protein ubiquitination in the presynapse, little is known about its regulation. The great distance of nerve terminals from the cell body poses a special problem for the control of protein turnover. The arrival of new material via axonal transport must continuously be balanced by either active retrograde axonal flow or local degradation. Membranous organelles and a variety of membrane-associated proteins are transported bidirectionally by "fast" axonal transport (23). However, pools of cytosolic proteins, which travel by the so-called "slow" axonal transport, move only anterogradally (24). The speed of slow transport is in the range of, at most, a few millimeters per day (24), so that proteins traveling by this mechanism may take weeks or even months to reach axon endings. Clearly, the degradation of these proteins must be blocked "en route" but then permitted in nerve terminals. Thus, proteolysis, including ubiquitin-dependent proteolyisis, must be finely regulated in axon endings.

If protein ubiquitination plays an important role in presynaptic function, this process is likely to be regulated by synaptic stimulation. Here, we have investigated whether depolarizationdependent $\mathrm{Ca}^{2+}$ entry affect the ubiquitinated state of synaptic proteins.

\section{Methods}

Antibodies, cDNAs, and Drugs. Antibodies directed against epsin 1, Eps15, amphiphysin 1, synapsin 1, and synaptophysin were generated in our labs and previously described $(25,26)$. Rabbit antibodies directed against amino acids 1476-1918 of mouse FAM were a kind gift of Kozo Kaibuchi (Nagoya University, Nagoya, Japan). Mouse monoclonal antiubiquitin antibodies were from Covance (Richmond, CA) and from Affinity, Nottingham, U.K. (FK2). Mouse monoclonal antibodies against the hemagglutinin (HA) epitope and $\beta$-tubulin were from Roche Applied Science (Indianapolis) and Sigma, respectively. Polyclonal antibodies directed against phosphosynapsin (phosphorylation site 3) were a gift from Andy Czernik, Angus Nairn, and Paul Greengard (The Rockefeller University, New York). Ionomycin, FK506, cyclosporin A and YU101 were purchased from Calbiochem. FAM cDNA was a kind gift from Stephen Wood (University of Adelaide, Adelaide, Australia).

Cell Culture. Cells were transfected with epsin 1 and HAubiquitin cDNAs in a pcDNA vector by using Lipofectamine (Invitrogen). Ionomycin (Calbiochem) was used at the concentration of either $1 \mu \mathrm{M}$ in serum-free DMEM supplemented with $1 \mathrm{mM} \mathrm{CaCl}_{2}$ (final $\left.\mathrm{Ca}^{2+}=2.3 \mathrm{mM}\right)\left(\mathrm{DMEM}^{*}\right)$ or of $6 \mu \mathrm{M}$ in DMEM $^{*}$ containing bovine serum $(10 \%)$. Cells were lysed either in $50 \mathrm{mM}$ Tris, pH $7.5 / 150 \mathrm{mM} \mathrm{NaCl} / 1 \mathrm{mM} \mathrm{MgCl}_{2} / 5 \mathrm{mM}$ EGTA/10 mM Na $3 \mathrm{VO}_{4} / 5 \mathrm{mM} N$-ethylmaleimide/protease inhibitor mixture (for liposome sedimentation experiments) or in the same buffer plus Triton X-100 (for the GST pulldowns of Fig. $5 B$ ) or in buffered $1 \%$ SDS also containing $1 \mathrm{mM} \mathrm{Na}_{3} \mathrm{VO}_{4}$ and immediately boiled for $10 \mathrm{~min}$. The latter material was then used for SDS/PAGE and Western blotting or further processed for immunoprecipitation or GST pulldowns (Fig. $5 C$ ). To this aim, SDS solubilized extracts were diluted with 9 volumes of $20 \mathrm{mM}$ Hepes, pH 7.4/50 mM NaCl/50 mM Na $3 \mathrm{PO}_{4} / 50 \mathrm{mM} \mathrm{NaF} / 5$ mM EDTA/5 mM EGTA/5 mM $N$-ethylmaleimide/1.1\% Triton $\mathrm{X}-100$ to scavenge SDS with excess Triton X-100. The resulting material was clarified by centrifugation at $14,000 \mathrm{rpm}$ $(20,000 \times g)$ for $10 \mathrm{~min}$ at $4^{\circ} \mathrm{C}$, and the supernatants were used for immunoprecipitations or GST pulldowns.

Small Interfering RNA (siRNA)-Mediated Suppression of FAM. Two pairs of oligonucleotides comprising mouse FAM sequences

Abbreviations: HA, hemagglutinin; DMEM*, serum-free DMEM supplemented with $1 \mathrm{mM}$ $\mathrm{CaCl}_{2}$; siRNA, small interfering RNA; CHO, Chinese hamster ovary.

ๆTo whom correspondence should be addressed. E-mail: pietro.decamilli@yale.edu.

() 2003 by The National Academy of Sciences of the USA 
182-200 (AAGATGAGGAACCTGCATTTC) and 3432-3452 (AAGGGGTGCCTACCTCAATGC) (which are 100\% conserved in human FAM) were generated (W.M. Keck Facility, Yale University, New Haven, CT). The oligonucleotides were deprotected by using tetrabutylammonium fluoride (Sigma) followed by desalting and annealing as described. HeLa cells were transfected with $50 \mathrm{nM}$ each of the two oligonucleotide pairs or $50 \mathrm{nM}$ of one pair of vimentin-specific oligonucleotides (a kind gift of Daiming Li, Yale University) by using Oligofectamine (Invitrogen) according to manufacturer's instructions and incubated for 3 days. Cells were then transfected with epsin 1 cDNA (25) in a pcDNA vector by using Lipofectamine and processed for immmunoprecipitations and Western blotting.

Synaptosome Experiments and Miscellaneous Procedures. Synaptosome preparation and stimulation were performed as described (26). In all cases, synaptosomes were used after a 10-min preincubation at $37^{\circ} \mathrm{C}$ to restore the metabolic state of living nerve terminals. Liposome sedimentation experiments were carried out as described (27). SDS/PAGE, Western blotting, immunoprecipitation, GST pulldowns, cell transfection, and immunofluorescence were performed according to standard procedures.

\section{Results}

To investigate protein ubiquitination in axon endings, we have used synaptosomes (pinched-off nerve terminals with attached fragments of postsynaptic elements), a model system commonly used for the study of neurosecretion and its regulation. Like intact synapses, synaptosomes can be stimulated by depolarization in high $\mathrm{K}^{+}$in the presence of $\mathrm{Ca}^{2+}$. This treatment also results in an increased state of phosphorylation of a variety of nerve terminal proteins, including synapsin $1(28-30)$, and in the dephosphorylation of other proteins $(26,29,31-34)$.

Antiubiquitin Western blotting of control synaptosomes revealed primarily the "smeary" pattern typical of polyubiquitinated proteins (Fig. 1A). Stimulation by depolarization in $50 \mathrm{mM}$ $\mathrm{K}^{+}$for $30 \mathrm{sec}$, i.e., a condition that results in massive evoked exocytic release of neurotransmitter $(35,36)$, induced a strong decrease in the overall levels of ubiquitinated proteins. This change required extracellular $\mathrm{Ca}^{2+}$ and correlated with the stimulation-dependent downward mobility shift of amphiphysin 1 (Fig. 1A), a clathrin accessory factor concentrated in nerve terminals. Like several other endocytic proteins, amphiphysin 1 is phosphorylated in resting nerve terminals and undergoes dephosphorylation on stimulation, with resulting increased mobility $(26,37)$.

The effect of stimulation on the state of protein ubiquitination (and on amphiphysin 1 dephosphorylation) was already nearly maximal at $15 \mathrm{sec}($ Fig. $1 B)$, the earliest time point tested. At 15 sec, an increase in the phosphorylation state of synapsin 1 (30), as demonstrated by Western blotting with an antiphosphosynapsin antibody (RU19) (Fig. 1B), was also observed, confirming the viability of synaptosomes and ATP availability under our experimental conditions. As in the case of the dephosphorylation of amphiphysin $(26,37)$ and other endocytic proteins (38), the decrease in protein ubiquitination was blocked by $0.5 \mu \mathrm{M}$ FK506 (Fig. $1 C$ ) or by $0.5 \mu \mathrm{M}$ cyclosporin A (not shown), two antagonists of the $\mathrm{Ca}^{2+}$-dependent phosphatase calcineurin (31, 39 ), and was reversed by the incubation of synaptosomes in control buffer for an additional $10 \mathrm{~min}$ (Fig. 1D). A further 30 -sec incubation in high $\mathrm{K}^{+}$, after the 10-min rest, triggered a new cycle of amphiphysin dephosphorylation and loss of protein ubiquitination (Fig. 1D). Loss of ubiquitination could in principle be explained, at least in part, by proteosomal degradation of polyubiquitinated proteins. However, a 30-min pretreatment of synaptosomes with the proteosomal inhibitor YU101 $(20 \mu \mathrm{M})$ did not inhibit the loss of ubiquitin immunoreactivity induced by
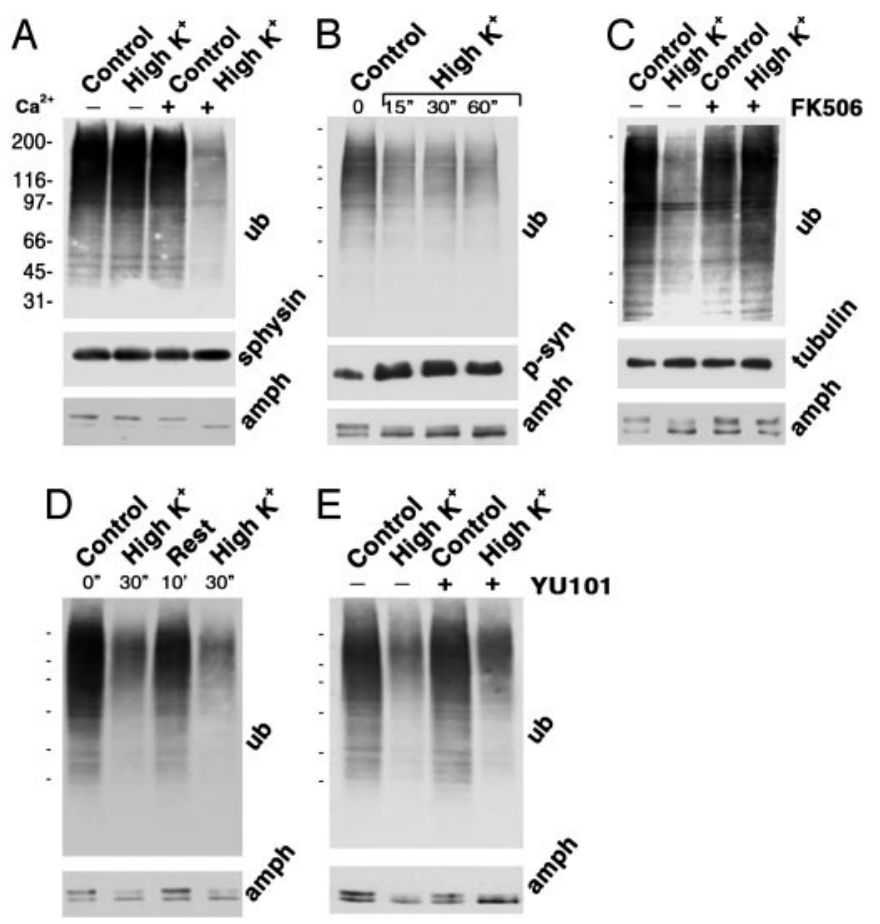

Fig. 1. Depolarization- and $\mathrm{Ca}^{2+}$-dependent change in protein ubiquitination at synapses. Rat-brain synaptosomes were preincubated for $10 \mathrm{~min}$ at $37^{\circ} \mathrm{C}$ in control physiological buffer, then further incubated at $37^{\circ} \mathrm{C}$ for $30 \mathrm{sec}$ (except for $B$ and $D$ ) under the conditions indicated. High $\mathrm{K}^{+}=50 \mathrm{mM} \mathrm{K}^{+}$with a corresponding decrease in $\mathrm{Na}^{+}$. For the zero $\mathrm{Ca}^{2+}$ condition of field $A, \mathrm{CaCl}_{2}$ was omitted and EGTA (1.2 mM final) was added. Incubations were stopped by SDS, and synaptosomal proteins were separated by SDS/PAGE and reacted by immunoblotting with antibodies to ubiquitin, amphiphysin, phosphosynapsin (antiphospho-site 3) (60), and, as a control for gel loading, synaptophysin, and tubulin. In $D$, the 30 -sec depolarization was followed by an additional $10 \mathrm{~min}$ in control buffer and finally by a second 30 -sec depolarization. Molecular weight standards are indicated in $A$ and simply as dashes in $B-E$. Sphysin, synaptophysin; $p$-syn, phosphosynapsin.

high $\mathrm{K}^{+}$(Fig. 1E). Thus, these changes must reflect either generalized deubiquitination or, most likely (see Discussion), impaired ubiquitination in the context of a very rapid ubiquitination-deubiquitination cycle.

The decrease in antiubiquitin immunoreactivity observed in stimulated synaptosomes affected both the "smear," thought to reflect polyubiquitination ladders, and the discrete bands. Because these bands may include monoubiquitinated proteins, we examined the ubiquitinated state of epsin 1 (25), a well characterized target of monoubiquitination $(6,40)$. Epsin 1 , which also binds ubiquitin via ubiquitin-interacting motif (UIM) domains (40), functions as an adaptor in clathrin coat assembly and may have additional roles in growth factor receptor signaling, actin regulation, and control of transcription (41-43). Like amphiphysin, epsin 1 undergoes $\mathrm{Ca}^{2+}$-dependent dephosphorylation in rat-brain synaptosomes (33), and this change is reflected in a downward mobility shift in SDS/PAGE (33). Antiubiquitin immunoblotting of antiepsin 1 immunoprecipitates revealed a pool of ubiquitinated epsin 1 in control synaptosomes and a $\mathrm{Ca}^{2+}$-dependent decrease of this pool in the stimulated samples (Fig. 2). Ubiquitinated epsin 1 migrated slightly slower than the bulk of epsin 1, did not undergo the lower mobility shift with stimulation, and was below detection in the antiepsin 1 immunoblot. Thus, ubiquitination is likely to involve only a very small fraction of total epsin 1. A major binding partner of epsin, Eps15 $(25,40)$, also undergoes monoubiquitination. As revealed by Western blotting of anti-Eps15 immunoprecipitates, a decrease 
A
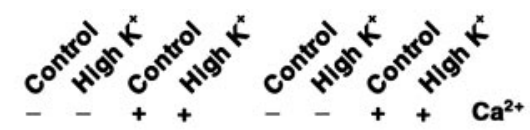

IP:epsin 1 WB:epsin 1
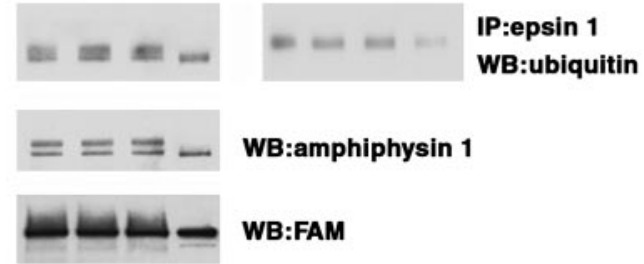

B

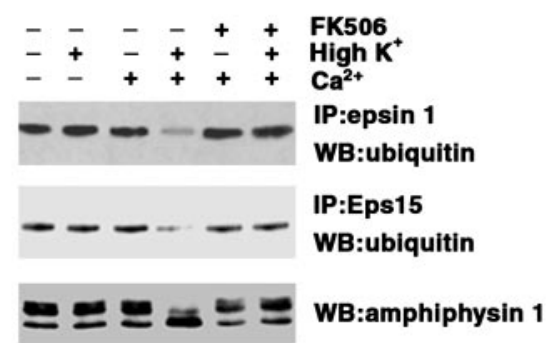

Fig. 2. Depolarization- and $\mathrm{Ca}^{2+}$-dependent decrease in the ubiquitinated state of epsin 1 and Eps15 at synapses. Rat-brain synaptosomes were preincubated for $10 \mathrm{~min}$ at $37^{\circ} \mathrm{C}$ in control buffer, then further incubated for $30 \mathrm{sec}$ at the same temperature in either control or high $\mathrm{K}^{+}$buffer and in the presence or absence of $\mathrm{Ca}^{2+}$ and of FK506 $(0.5 \mu \mathrm{M})$ and finally solubilized in SDS. This material was directly processed for SDS/PAGE and Western blotting (WB) for amphiphysin 1 and FAM, or reacted with excess Triton X-100 (to remove free SDS) and subjected to antiepsin 1 and anti-Eps15 immunoprecipitation (IP). Immunoprecipitates were then analyzed by SDS/PAGE and immunoblotting for epsin 1, Eps15, and ubiquitin. The two blots at $A$ Top are from the same gel and are precisely aligned to show slight differences in migration between the bulk of epsin 1 immunoreactivity (Left) and ubiquitinated epsin 1 (Right).

in the state of ubiquitination occurred for this protein as well in stimulated synaptosomes (Fig. 2B). FK506 not only blocked the dephosphorylation of amphiphysin 1 (ref. 26 and Fig. $2 B$ ), epsin 1 (33), and Eps15 (33), but also inhibited the decrease of ubiquitinated epsin 1 and Eps15 (Fig. 2B).

In Drosophila, epsin binds to, and is a physiological substrate for, the deubiquitinating enzyme fat facets and is a critical mediator of the function of this enzyme in development $(44,45)$. Overexpression of fat facets in neurons affects synaptic morphology (18). Whether fat facets is normally present at synapses is not known. We have now found (i) that antibodies directed against epsin 1 and FAM, the mammalian homologue of fat facets (46), produce an overlapping immunostaining of synapses in frozen sections of rat brain (Fig. 3A); (ii) that FAM coprecipitates with anti-epsin 1 immunoprecipitates (Fig. 3B); and (iii) that epsin 1 is specifically retained by an immobilized fusion protein of the catalytic domain of FAM (Fig. 3C). Western blotting of purified synaptosomes with anti-FAM antibodies revealed a prominent broad band that became sharper after stimulation, possibly reflecting $\mathrm{Ca}^{2+}$-dependent changes (Fig. $2 A$ ) such as dephosphorylation. Accordingly, preliminary evidence suggests that the interaction between epsin 1 and FAM may be negatively regulated by phosphorylation (not shown).

We further investigated the role of FAM in the deubiquitination of epsin 1 by using nonneuronal cells [Chinese hamster ovary (CHO) and HeLa cells], where FAM expression could be suppressed by siRNA. We first characterized the ubiquitination of epsin 1 in these cells. Cells were transfected with epsin 1 and in some case also with HA-tagged ubiquitin, thus allowing the analysis of the ubiquitinated state of epsin 1 by using anti-HA antibodies, a very sensitive detection method. Although only one
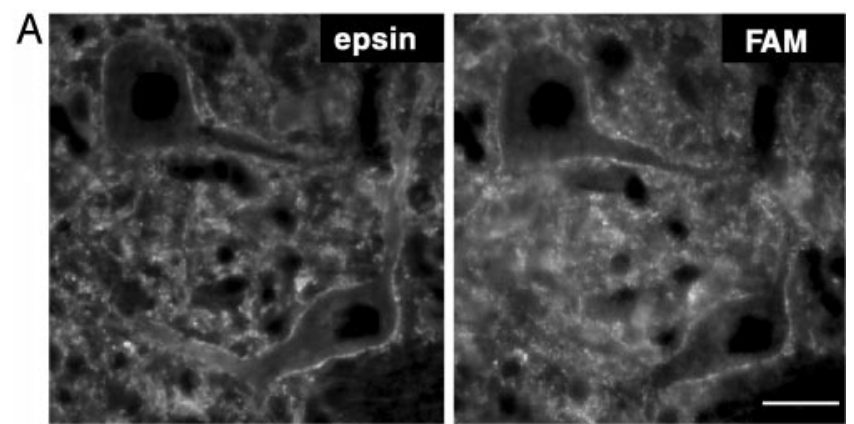

B
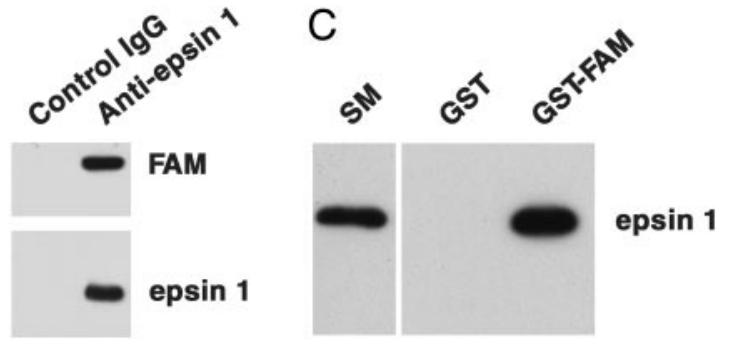

Fig. 3. Colocalization and interaction of epsin 1 with FAM in brain. (A) Double immunofluorescence of rat-brain frozen sections. (Bar $=30 \mu \mathrm{m}$.) $(B)$ Control or anti-epsin 1 immunoprecipitates generated from rat-brain cytosol were immunoblotted for either FAM or epsin 1, thus revealing coprecipitation of the two proteins. (C) Anti-epsin 1 immunoblot of material affinity-purified from brain (SM, starting material) by GST or a GST fusion protein of the catalytic domain of mouse FAM (amino acids 1554-1953).

ubiquitinated epsin 1 band was observed after transfection of epsin 1 alone (Fig. $4 A$ and $B$ ), multiple ubiquitinated epsin 1 species were observed in HA-ubiquitin transfected cells (Fig. 4 $C-F$ ), possibly reflecting multimonoubiquitination (47) due to ubiquitin overexpression. The ubiquitinated pool of epsin was detected only by antibodies that recognize ubiquitin (anti-HA antibodies) and not by antiepsin antibodies (Fig. 4C). Thus, as observed at synapses, ubiquitination involves only a very small pool of the protein, although a contribution to this result of epitope masking by multiple ubiquitination cannot be excluded.

$\mathrm{Ca}^{2+}$ influx into nonneuronal cells was induced by the $\mathrm{Ca}^{2+}$ ionophore ionomycin in the presence of $2.3 \mathrm{mM}$ extracellular $\mathrm{Ca}^{2+}$. As a control for the occurrence of $\mathrm{Ca}^{2+}$-stimulated reactions, the electrophoretic mobility of cotransfected amphiphysin 1 was monitored, because even in nonneuronal cells, this protein undergoes dephosphorylation in response to $\mathrm{Ca}^{2+}$ influx (S. Floyd and P.D.C., unpublished observations and Fig. $4 A$ ). FAM, which in well resolved gels migrated as a doublet, collapsed into a single lower band in ionomycin-treated $\mathrm{CHO}$ cells (Fig. $4 A$ ), possibly reflecting $\mathrm{Ca}^{2+}$-dependent dephosphorylation. Ionomycin also produced a decrease in the global ubiquitinated state of proteins in both $\mathrm{CHO}$ and HeLa cells (not shown and Fig. $4 G$ ) as well as a decrease of either endogenous (Fig. $4 A$ and $B$ ) or HA-tagged ubiquitin (Fig. $4 C$ ) associated with wild-type epsin 1 , as detected by Western blots of antiepsin 1 immunoprecipitates (Fig. $4 A$ and $C$ ). These changes could already be observed $1 \mathrm{~min}$ after the addition of $1 \mu \mathrm{M}$ ionomycin (Fig. $4 D$ ) and were reversed by removal of ionomycin and further incubation with control medium for $10 \mathrm{~min}$ (Fig. $4 B$ ). The ionomycin-induced effect in HA-ubiquitin transfected cells was not inhibited by pretreatment of cells for $3 \mathrm{~h}$ with the proteasome inhibitor YU101 (Fig. $4 E$ ) but was blocked by FK506 (Fig. $4 F$ ), consistent with a change in the ubiquitinated state, rather than with an effect of protein degradation. As in the case of synaptosomes, inhibition of an upstream step in the ubiquitination 


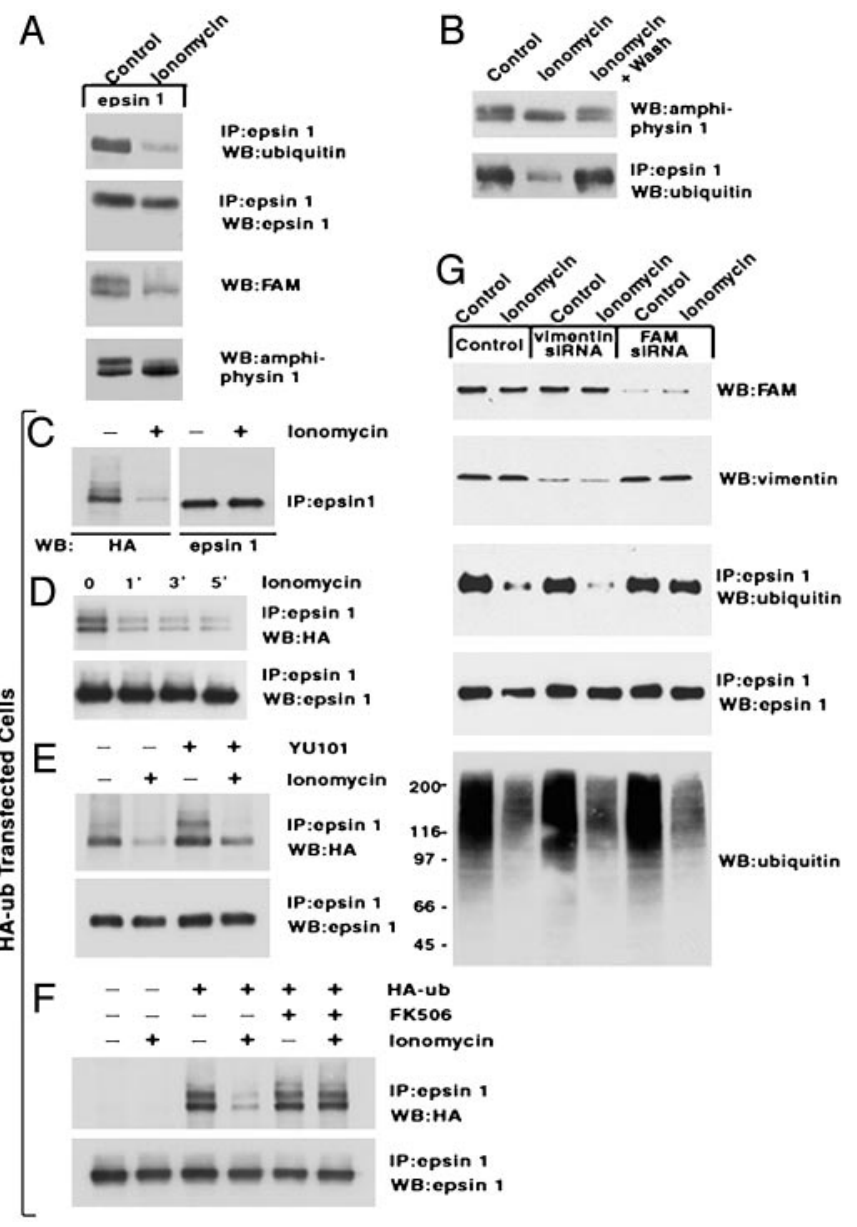

Fig. 4. $\mathrm{Ca}^{2+}$-dependent deubiquitination of epsin in nonneuronal cells and role of FAM in this reaction. Cells were subjected to various treatments, then homogenized and either subjected to immunoprecipitation (IP) followed by SDS/PAGE and Western blotting (WB) (in the case of epsin 1) or directly processed by SDS/PAGE and Western blotting (in the case of other proteins). $(A$ and $B)$ CHO cells were cotransfected with amphiphysin 1 and epsin 1 . Transfected cells were incubated in DMEM* (total $\mathrm{Ca}^{2+}=2.3 \mathrm{mM}$, see Methods) and $10 \%$ FBS in the absence or presence of ionomycin ( $6 \mu \mathrm{M})$ for $15 \mathrm{~min}$ $(A)$ or $5 \mathrm{~min}(B)$. In the case of $B$, a sample of ionomycin-treated cells was then returned to ionomycin-free medium for $10 \mathrm{~min}$ (right lane). (C) $\mathrm{CHO}$ cells cotransfected with HA-ubiquitin and epsin 1 were incubated for 5 min in DMEM* in the presence or absence of ionomycin (1 $\mu \mathrm{M})$. Bands that most likely reflect mono-, di-, and triubiquitinated epsin (Left) migrate slightly above the bulk of epsin immunoreactivity (Right). (D) CHO cells cotransfected with HA-ubiquitin and epsin 1 were incubated for the time indicated in DMEM*. $(E)$ $\mathrm{CHO}$ cells cotransfected with HA-ubiquitin and epsin 1 were incubated for $3 \mathrm{~h}$ in DMEM* plus the proteasome inhibitor YU101 and then incubated for an additional 5 min in DMEM* in the absence or presence of ionomycin (1 $\mu \mathrm{M})$. Although the level of ubiquitinated epsin 1 is increased by inhibition of the proteasome, the effect of ionomycin is not inhibited. $(F) \mathrm{CHO}$ cells transfected with epsin 1 or cotransfected with both epsin 1 and HA-ubiquitin were incubated for $5 \mathrm{~min}$ with and without ionomycin $(1 \mu \mathrm{M})$ and with or without FK506 $(0.5 \mu \mathrm{M})$, as indicated. (G) HeLa cells were incubated for 3 days in control conditions with two pairs of FAM-specific siRNAs or one pair of vimentinspecific siRNAs as an additional control. They were then transfected with epsin 1 in the continued presence of the siRNAs. Twenty-four hours after transfection, cells were exposed to ionomycin stimulation as in $C$, then analyzed by immunoprecipitation and Western blotting.

cascade appears as the more likely explanation for ionomycin effect.

siRNA experiments were performed in HeLa cells. A 3-d incubation with siRNAs specific for FAM or for the control protein vimentin strongly inhibited endogenous FAM or vimen- tin expression, respectively (Fig. $4 G$ ). In both sets of cells, the general decrease of protein ubiquitination induced by ionomycin-mediated $\mathrm{Ca}^{2+}$ influx was still observed, but in the FAMsiRNA-treated samples, the loss of ubiquitin from epsin 1 was selectively inhibited (Fig. 4G). These findings demonstrate a role of FAM in epsin 1 deubiquitination, in agreement with studies of the Drosophila homologues of these two proteins (45), but not in the global decrease in the ubiquitination state of proteins. This global decrease is likely to include a large variety of deubiquitinating enzymes with different substrate specificities.

Epsin acts as a multifunctional adaptor in endocytic traffic via its interaction with phosphatidylinositol 4,5-bisphosphate $\left[\mathrm{PI}(4,5) \mathrm{P}_{2}\right]$, clathrin, the clathrin adaptor AP-2, Eps15, and possibly ubiquitinated membrane proteins $(42,43)$. It was of interest to determine whether the ubiquitination of epsin affects its binding properties. Due to the lability of ubiquitination in tissue and cell extracts, we could not reliably analyze the interaction of epsin from brain tissue or untransfected cells. We used extracts of cells cotransfected with HA-ubiquitin and epsin 1 , where a greater pool of epsin is in the ubiquitinated form and where the HA epitope facilitates ubiquitin detection. To test the interaction with lipids, a cell lysate was incubated with synthetic liposomes comprising $70 \%$ phosphatidylcholine, $20 \%$ phosphatidylserine (PS), and either $10 \% \mathrm{PI}(4,5) \mathrm{P}_{2}$ or an additional $10 \%$ PS. Liposome-bound epsin 1 was recovered by centrifugation, followed by solubilization of the pellet in SDS, addition of Triton X-100 to titrate out SDS, antiepsin immunoprecipitation, and finally Western blotting for either epsin 1 or ubiquitin. Epsin 1 was efficiently recovered on $\mathrm{PI}(4,5) \mathrm{P}_{2}$-containing liposomes, with a corresponding decrease in the supernatant. However, ubiquitinated epsin was not enriched on liposomes, irrespective of the presence of $\mathrm{PI}(4,5) \mathrm{P}_{2}$ (Fig. $5 A$ ). Likewise, pulldown assays with GST fusion proteins of the ear domain of the clathrin adaptor AP-2 and of the $\mathrm{NH}_{2}$-terminal domain of clathrin, i.e., the epsin interacting regions of these two coat proteins $(48,49)$, revealed a very prominent affinity purification of epsin 1 (with a partial depletion from the unbound material) but not of HA-ubiquitin-tagged epsin 1 (Fig. $5 B$ and $C$ ) or other HAtagged proteins (Fig. 5B). In contrast, a GST fusion protein of the Eps15 homology domain-containing region of Eps15 pulled down ubiquitinated epsin 1 (Fig. $5 C$ ).

The ubiquitinated state of epsin 1 produced by overexpression of HA-ubiquitin may not reflect its normal ubiquitinated state. Thus, it remains to be determined whether loss of the interactions with liposomes, clathrin, and AP-2 revealed by these experiments are physiologically relevant. Furthermore, ubiquitinated epsin 1 may also be phosphorylated, and phosphorylation may contribute to the loss of some interactions. However, the nearly complete lack of HA-ubiquitin immunoreactivity on the material bound to clathrin and AP-2 contrasts with the much less pronounced inhibition of binding observed for phosphorylated epsin 1 (33). An interesting possibility is that the ubiquitin covalently bound to epsin 1 may interact with epsin's ubiquitininteracting motif domain, thus forming an intramolecular interaction that occludes the binding sites for $\mathrm{PI}(4,5) \mathrm{P}_{2}$, clathrin, and AP-2 but not the binding sites for Eps15 homology domains, which are localized in the $\mathrm{COOH}$-terminal region of the protein.

\section{Discussion}

We report here that depolarization-dependent $\mathrm{Ca}^{2+}$ influx induces a very rapid and general decrease of the ubiquitinated state of synaptic proteins, including monoubiquitinated proteins. The fast kinetics of this change and its insensitivity to a proteasome inhibitor suggest deubiquitination rather than proteosomal degradation. Results from nonneuronal cells, where similar effects were produced by ionomycin-induced $\mathrm{Ca}^{2+}$ entry, demonstrate that disruption of a single deubiquitinating enzyme, 
A

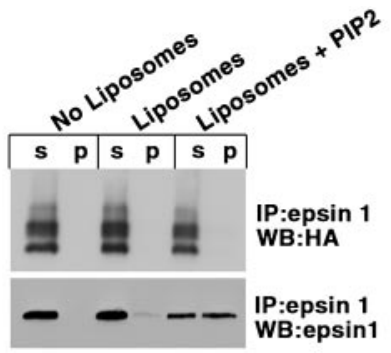

B
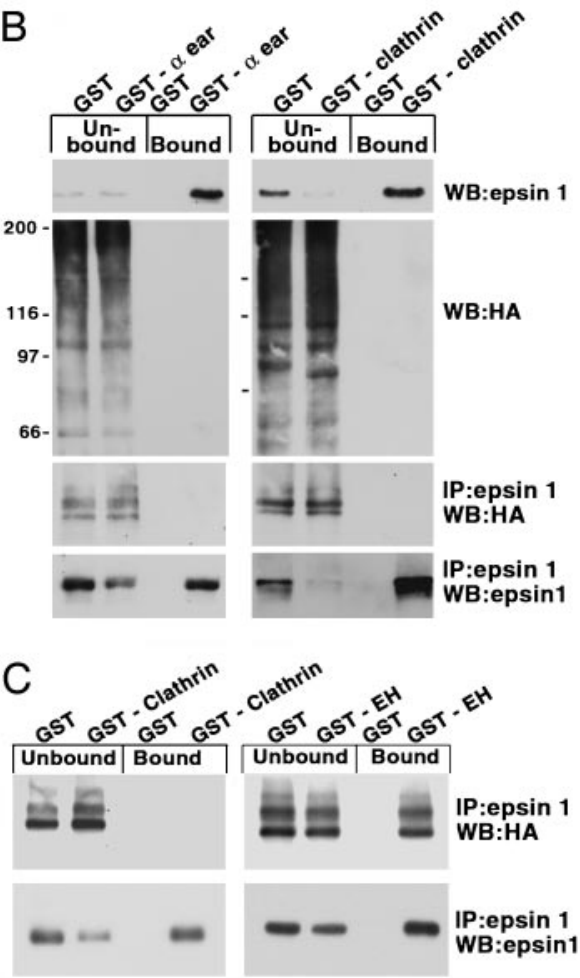

Fig. 5. Ubiquitination of epsin 1 in cells overexpressing HA-ubiquitin inhibits its interaction with liposomes, clathrin, and AP-2, but not Eps15. CHO cells were cotransfected with HA-ubiquitin and epsin 1. (A) A cytosolic fraction (prepared by centrifugation of cell extracts not containing Triton X-100) was incubated with liposomes for $30 \mathrm{~min}$ at $37^{\circ} \mathrm{C}$, and liposome-bound material was recovered by sedimentation. Supernatants (s) and pellets ( $p)$ were then subjected to immunoprecipitation and Western blotting to reveal HAubiquitin and epsin 1. ( $B$ and $C$ ) Triton X-100 solubilized cell extracts $(B)$ or cell extracts obtained by SDS solubilization followed by Triton treatment $(C)$ were affinity-purified on glutathione-immobilized GST or GST fusions of the ear domain of AP-2 of the $\mathrm{NH}_{2}$-terminal region of clathrin heavy chain and of the Eps15 homology domain region of Eps15. The presence of HA-ubiquitin and epsin 1 in the unbound and bound material was then revealed by Western blotting either without further processing or after anti-epsin 1 immunoprecipitation, as indicated.

FAM (46), blocks the deubiquitination of epsin 1 but not global deubiquitination.

In principle, $\mathrm{Ca}^{2+}$ could produce a general stimulation of deubiquitinating enzymes. However, given the multiplicity of deubiquitinating enzymes, a general stimulation of deubiquitination reactions seems unlikely. Deubiquitinating enzymes are known to be very active. Thus, an alternative possibility is that rapid deubiquitination (including deubiquitination mediated by FAM) occurs constitutively and that $\mathrm{Ca}^{2+}$ inhibits some upstream step(s) in the chain of reactions leading to protein ubiquitination. In fact, rapid loss of protein-associated ubiquitin was observed under conditions that deplete synaptosomal ATP (incubation with the mitochondrial uncoupler FCCP and deoxyglucose $(50,51)]$ (unpublished observations), in agreement with the ATP dependence of protein ubiquitination. The high $\mathrm{K}^{+}$ stimulation protocol used, however, did not produce a drop of ATP levels (52) (unpublished observations), and the stimulation-dependent deubiquitination occurred concomitantly to the phosphorylation of synapsin 1. Furthermore, the conditions shown here to induce global deubiquitination are the same as those known to stimulate exocytic release of neurotransmitter, an ATP-dependent reaction $(35,36)$. Thus, if the effects observed are, at least in part, due to inhibition of ubiquitination, they are likely to result from the $\mathrm{Ca}^{2+}$-dependent inhibition of specific biochemical reactions. Irrespective of the mechanisms responsible for the observed changes, our results demonstrate a remarkably rapid turnover of ubiquitin in synaptosomes.

The stimulated decrease in protein ubiquitination occurs in parallel to the dephosphorylation of a variety of synaptic proteins, primarily endocytic proteins including dynamin, amphiphysin, epsin 1, and Eps15 (26, 31-33). Both processes are blocked by FK506 and cyclosporin A, two inhibitors of the $\mathrm{Ca}^{2+}$-dependent phosphatase calcineurin (39), suggesting an involvement of this enzyme. Calcineurin was shown to have proapoptotic effects $(53,54)$, yet the events described here are too fast to be accounted for by this action and are rapidly reversible. One must also consider the possibility that not all of the effects of FK506 and cyclosporin A are mediated by calcineurin antagonism via their interactions with FKBP12 and cyclophillin, respectively $(39,55)$. However, rapamycin that forms a complex with FKBP12 that does not inhibit calcineurin (39) did not inhibit $\mathrm{Ca}^{2+}$-dependent deubiquitination (not shown).

The precise physiological significance of the "global" regulation of ubiquitination revealed by our study remains to be determined. As discussed in the Introduction, protein-mediated degradation must be finely tuned in axon terminals. $\mathrm{A} \mathrm{Ca}^{2+}$ dependent decrease in the steady-state level of protein ubiquitination may provide a feedback mechanism between synaptic activity and the rate of protein degradation, thus leading to the stabilization of active nerve terminals. Proteasome inhibitors were shown to enhance neurite outgrowth and synaptic strength $(13,56,57)$. It will be of interest to determine the interplay of $\mathrm{Ca}^{2+}$-regulated ubiquitin-dependent proteasomal degradation with the $\mathrm{Ca}^{2+}$ /calpain-dependent breakdown (58) of a variety of synaptic proteins.

The strong decrease of the ubiquitinated state of synaptic proteins after depolarization-dependent $\mathrm{Ca}^{2+}$ entry contrasts with the enhanced rate of protein ubiquitination and degradation observed by Ehlers (11) in neuronal cultures chronically stimulated by the block of inhibitory neurotransmission. One difference between the two studies is that Ehlers focused selectively on postsynaptic proteins, whereas our synaptosomes studies are likely to favor the detection of changes in the presynaptic compartment. A more important difference between the two studies, however, is represented by the two modes of stimulation, acute in our case and chronic in the case of Ehlers. There are other examples of opposite actions produced by an acute $\mathrm{Ca}^{2+}$ influx vs. prolonged and modulatory stimuli. For instance, phosphorylation of Eps15 and epsin is enhanced by growth factor receptor signals (59) (unpublished results), whereas an acute rise of cytosolic $\mathrm{Ca}^{2+}$ induces dephosphorylation of both proteins (33). Moreover, both Eps15 and epsin 1 undergo an increase in their ubiquitinated state in response to growth factors (40), in contrast to the rapid $\mathrm{Ca}^{2+}$-dependent deubiquitination reported here.

Nerve terminal stimulation activates the synaptic vesicle cycle and therefore endocytosis. Because mono- and oligoubiquination were shown to regulate components of the endocytic pathway $(5,6)$, changes in the steady-state level of 
protein ubiquitination may also be linked to changes in membrane traffic. In the case of epsin 1, evidence obtained from cells overexpressing ubiquitin and epsin 1 (but it remains to be seen whether this observation also applies to epsin ubiquitinated under more physiological conditions) suggests that ubiquitination impairs its clathrin adaptor functions. Thus, a stimulationdependent deubiquitination of epsin 1 would be consistent with an enhanced rate of endocytosis. However, only an extremely small pool of epsin appears to be involved in ubiquitinationdeubiquitination reactions at any given time in synaptosomes, speaking against the possibility that ubiquitination may have a major role in keeping epsin 1 in a nonassembled state in resting nerve terminals. Furthermore, ubiquitination is thought to correlate with activation of the endocytic pathway (4-6) rather than with its resting state. Ubiquitinated epsin may represent a very transient intermediate, possibly involved in generating a specific

1. Hochstrasser, M. (1996) Annu. Rev. Genet. 30, 405-439.

2. Hershko, A. \& Ciechanover, A. (1998) Annu. Rev. Biochem. 67, 425-479.

3. Pickart, C. M. (2001) Annu. Rev. Biochem. 70, 503-533.

4. Katzmann, D. J., Odorizzi, G. \& Emr, S. D. (2002) Nat. Rev. Mol. Cell Biol. 3, 893-905.

5. Hicke, L. (2001) Nat. Rev. Mol. Cell Biol. 2, 195-201.

6. Aguilar, R. C. \& Wendland, B. (2003) Curr. Opin. Cell Biol. 15, 184-190.

7. Wheeler, T. C., Chin, L. S., Li, Y., Roudabush, F. L. \& Li, L. (2002) J. Biol. Chem. 277, 10273-10282.

8. Chin, L. S., Vavalle, J. P. \& Li, L. (2002) J. Biol. Chem. 277, 35071-35079.

9. Shimura, H., Schlossmacher, M. G., Hattori, N., Frosch, M. P., Trockenbacher, A., Schneider, R., Mizuno, Y., Kosik, K. S. \& Selkoe, D. J. (2001) Science 293, 263-269.

10. Burbea, M., Dreier, L., Dittman, J. S., Grunwald, M. E. \& Kaplan, J. M. (2002) Neuron 35, 107-120.

11. Ehlers, M. D. (2003) Nat. Neurosci. 6, 231-242.

12. Hegde, A. N., Inokuchi, K., Pei, W., Casadio, A., Ghirardi, M., Chain, D. G., Martin, K. C., Kandel, E. R. \& Schwartz, J. H. (1997) Cell 89, 115-126.

13. Zhao, Y., Hegde, A. N. \& Martin, K. C. (2003) Curr. Biol. 13, 887-898.

14. Speese, S. D., Trotta, N., Rodesch, C. K., Aravamudan, B. \& Broadie, K. (2003) Curr. Biol. 13, 899-910.

15. Campbell, D. S. \& Holt, C. E. (2001) Neuron 32, 1013-1026.

16. Myat, A., Henry, P., McCabe, V., Flintoft, L., Rotin, D. \& Tear, G. (2002) Neuron 35, 447-459.

17. Watts, R. J., Hoopfer, E. D. \& Luo, L. (2003) Neuron 38, 871-885.

18. DiAntonio, A., Haghighi, A. P., Portman, S. L., Lee, J. D., Amaranto, A. M \& Goodman, C. S. (2001) Nature 412, 449-452.

19. Wilson, S. M., Bhattacharyya, B., Rachel, R. A., Coppola, V., Tessarollo, L., Householder, D. B., Fletcher, C. F., Miller, R. J., Copeland, N. G. \& Jenkins, N. A. (2002) Nat. Genet. 32, 420-425.

20. Giasson, B. I. \& Lee, V. M. (2003) Cell 114, 1-8.

21. Shimura, H., Hattori, N., Kubo, S., Mizuno, Y., Asakawa, S., Minoshima, S., Shimizu, N., Iwai, K., Chiba, T., Tanaka, K. \& Suzuki, T. (2000) Nat. Genet. 25, 302-305.

22. Taylor, J. P., Hardy, J. \& Fischbeck, K. H. (2002) Science 296, 1991-1995.

23. Hirokawa, N. (1997) Curr. Opin. Neurobiol. 7, 605-614.

24. Lasek, R. T. \& Hoffman, P. N. (1976) in Cell Motility, eds. Goldman, R., Pollard, T. D. \& Rosenbaum, J. (Cold Spring Harbor Lab. Press, Plainview, NY), Vol. 3, pp. 1021-1049.

25. Chen, H., Fre, S., Slepnev, V. I., Capua, M. R., Takei, K., Butler, M. H., Di Fiore, P. P. \& De Camilli, P. (1998) Nature 394, 793-797.

26. Bauerfeind, R., Takei, K. \& De Camilli, P. (1997) J. Biol. Chem. 272, 30984-30992.

27. Wenk, M. \& De Camilli, P. (2003) Methods Enzymol. 372, 248-260.

28. Schulman, H. \& Greengard, P. (1978) Nature 271, 478-479.

29. Wang, J. K., Walaas, S. I. \& Greengard, P. (1988) J. Neurosci. 8, 281-288.

30. De Camilli, P., Benfenati, F., Valtorta, F. \& Greengard, P. (1990) Annu. Rev. Cell Biol. 6, 433-460. conformation of this protein, via intramolecular interactions between ubiquitin and the ubiquitin-interacting motif domain.

In conclusion, synaptic stimulation has dramatic effects on protein ubiquitination. Genetic evidence demonstrates that abnormal protein ubiquitination may play a role in diseases of the nervous system $(9,19,21,22)$. The further elucidation of mechanisms in protein ubiquitination at the synapse is expected to provide new information of significant interest in synaptic physiology, general cell biology, and medicine.

We thank Huaqing Cai for help in some of the initial siRNA experiments; Scott Floyd for discussing preliminary results; and Hemmo Meyer, Graham Warren, and Mark Hochstrasser for discussion. This work was supported in part by a Human Frontiers Science Program grant (to P.D.C. and P.P.D.F.), and by grants from the National Institutes of Health (to P.D.C.), and from Associazione Italiana Ricerca sul Cancro, the Telethon Foundation, and the European Community (to P.P.D.F.)

31. Liu, J. P., Sim, A. T. \& Robinson, P. J. (1994) Science 265, 970-973.

32. Slepnev, V. I., Ochoa, G. C., Butler, M. H., Grabs, D. \& Camilli, P. D. (1998) Science 281, 821-824.

33. Chen, H., Slepnev, V. I., Di Fiore, P. P. \& De Camilli, P. (1999) J. Biol. Chem. 274, 3257-3260.

34. Cousin, M. A., Tan, T. C. \& Robinson, P. J. (2001) J. Neurochem. 76, 105-116. 35. Nicholls, D. G. \& Sihra, T. S. (1986) Nature 321, 772-773.

36. Marks, B. \& McMahon, H. T. (1998) Curr. Biol. 8, 740-749.

37. Slepnev, V. I. \& De Camilli, P. (2000) Nat. Rev. Neurosci. 1, 161-172.

38. Cousin, M. A. \& Robinson, P. J. (2001) Trends Neurosci. 24, 659-665.

39. Schreiber, S. L. \& Crabtree, G. R. (1992) Immunol. Today 13, 136-142.

40. Polo, S., Sigismund, S., Faretta, M., Guidi, M., Capua, M. R., Bossi, G., Chen, H., De Camilli, P. \& Di Fiore, P. P. (2002) Nature 416, 451-455.

41. Di Fiore, P. P., Pelicci, P. G. \& Sorkin, A. (1997) Trends Biochem. Sci. 22, 411-413.

42. Wendland, B. (2002) Nat. Rev. Mol. Cell Biol. 3, 971-977.

43. De Camilli, P., Chen, H., Hyman, J., Panepucci, E., Bateman, A. \& Brunger, A. T. (2002) FEBS Lett. 513, 11-18.

44. Cadavid, A. L., Ginzel, A. \& Fischer, J. A. (2000) Development (Cambridge, U.K) 127, 1727-1736

45. Chen, X., Zhang, B. \& Fischer, J. A. (2002) Genes Dev. 16, 289-294.

46. Taya, S., Yamamoto, T., Kanai-Azuma, M., Wood, S. A. \& Kaibuchi, K. (1999) Genes Cells 4, 757-767.

47. Haglund, K., Sigismund, S., Polo, S., Szymkiewicz, I., Di Fiore, P. P. \& Dikic, I. (2003) Nat. Cell Biol. 5, 461-466.

48. Rosenthal, J. A., Chen, H., Slepnev, V. I., Pellegrini, L., Salcini, A. E., Di Fiore, P. P. \& De Camilli, P. (1999) J. Biol. Chem. 274, 33959-33965.

49. Drake, M. T., Downs, M. A. \& Traub, L. M. (2000) J. Biol. Chem. 275, 6479-6489.

50. Akerman, K. E. \& Nicholls, D. G. (1981) FEBS Lett. 135, 212-214.

51. Pocock, J. M. \& Nicholls, D. G. (1998) J. Neurochem. 70, 806-813.

52. Erecinska, M., Nelson, D. \& Chance, B. (1991) Proc. Natl. Acad. Sci. USA 88, $7600-7604$

53. Wang, H. G., Pathan, N., Ethell, I. M., Krajewski, S., Yamaguchi, Y., Shibasaki, F., McKeon, F., Bobo, T., Franke, T. F. \& Reed, J. C. (1999) Science 284, 339-343.

54. Springer, J. E., Azbill, R. D., Nottingham, S. A. \& Kennedy, S. E. (2000) J. Neurosci. 20, 7246-7251.

55. Snyder, S. H., Lai, M. M. \& Burnett, P. E. (1998) Neuron 21, 283-294.

56. Saito, Y. \& Kawashima, S. (1989) J. Biochem. (Tokyo) 106, 1035-1040.

57. Ohtani-Kaneko, R., Takada, K., Iigo, M., Hara, M., Yokosawa, H., Kawashima, S., Ohkawa, K. \& Hirata, K. (1998) Neurochem. Res. 23, 1435-1443.

58. Chan, S. L. \& Mattson, M. P. (1999) J. Neurosci. Res. 58, 167-190.

59. Fazioli, F., Minichiello, L., Matoskova, B., Wong, W. T. \& Di Fiore, P. P. (1993) Mol. Cell. Biol. 13, 5814-5828.

60. Czernik, A. J., Girault, J. A., Nairn, A. C., Chen, J., Snyder, G., Kebabian, J. \& Greengard, P. (1991) Methods Enzymol. 201, 264-283. 